Portland State University

PDXScholar

6-1-1978

\title{
Existence of the Dielectric Constant in Fluids of Nonlinear Rigid Polar Molecules
}

John D. Ramshaw

Portland State University, jdramshaw@yahoo.com

Follow this and additional works at: https://pdxscholar.library.pdx.edu/phy_fac

Part of the Physics Commons

Let us know how access to this document benefits you.

\section{Citation Details}

J.D. Ramshaw, "Existence of the dielectric constant in fluids of nonlinear rigid polar molecules," J. Chem. Phys. 68, 5199 (1978)

This Article is brought to you for free and open access. It has been accepted for inclusion in Physics Faculty Publications and Presentations by an authorized administrator of PDXScholar. Please contact us if we can make this document more accessible: pdxscholar@pdx.edu. 


\title{
Existence of the dielectric constant in fluids of nonlinear rigid polar molecules ${ }^{a)}$
}

\author{
John D. Ramshaw \\ Theoretical Division, University of California, Los Alamos Scientific Laboratory, Los Alamos, New \\ Mexico 87545 \\ (Received 5 January 1978)
}

\begin{abstract}
The existence of the dielectric constant $\epsilon$ is investigated for fluids composed of nonlinear rigid polar molecules. The investigation is performed using the functional-derivative approach previously employed to establish sufficient conditions for the existence of $\epsilon$ in fluids of linear (axially symmetric) molecules. It is shown that these same conditions are sufficient for nonlinear molecules of arbitrary symmetry. An expression for $\epsilon$ in terms of the direct correlation function emerges automatically from the development. This expression, which involves the inversion of a $3 \times 3$ matrix, is a slight generalization of one obtained earlier by Hoye and Stell using an entirely different approach.
\end{abstract}

\section{INTRODUCTION}

This article is the fourth in a series concerned with dielectric behavior and pair correlations in fluids composed of rigid (unpolarizable) polar molecules. As in the previous articles, ${ }^{1-3}$ our primary concern is with the conditions under which the dielectric constant $\epsilon$ may be shown to exist. Attention was previously restricted to linear (axially symmetric) molecules, so that the essential features of the problem could be identified and clarified in the absence of peripheral complications. It was discovered that the direct correlation function $c(12)$ is of central importance to dielectric behavior, and that sufficient conditions for the existence of $\epsilon$ could be simply expressed in terms of it. ${ }^{2}$ The least restrictive such conditions were obtained ${ }^{3}$ by exploiting the functional-derivative interpretation of $c(12)$ in terms of an inverse response kernel. ${ }^{4}$

The purpose of the present article is to extend this previous work to nonlinear molecules of arbitrary symmetry. The development follows the same outline as that of the axially symmetric case, ${ }^{3}$ but is naturally somewhat more complicated in its details. However, the final sufficient conditions for the existence of $\epsilon$ are the same as before: $\epsilon$ exists if $c(12)$ is the sum of a short-range term that depends only on relative positions and orientations of molecules 1 and 2, and the longrange term $-\phi_{d}(12) / k T$ (where $\phi_{d}$ is the dipole-dipole potential).

As usual, an expression for $\epsilon$ in terms of $c(12)$ emerges automatically from the development. In contrast to the simplicity of the axially symmetric case, this expression involves the inverse of a $3 \times 3$ matrix whose elements are formed from spatial integrals of various angular moments of $c(12)$. This expression is a slight generalization of one derived earlier by $\mathrm{H} \phi y e$ and Stell ${ }^{5}$ using an entirely different approach. (Høye and Stell derived their expressions for $\epsilon$ by considering electrostatic interactions in the interior of an infinite sample. ${ }^{5,8}$ They were not specifically concerned with

\footnotetext{
a) Work performed in part under the auspices of the United States Department of Energy.
}

the existence of $\epsilon$, the investigation of which requires one to consider a finite sample of arbitrary shape in a nonuniform external electric field. ${ }^{1}$ )

\section{THE EXISTENCE OF $\epsilon$}

Consider a finite volume $V$, of arbitrary shape, containing $N$ identical rigid polar molecules of arbitrary symmetry. The number density $N / V$ is denoted by $\rho$. The position and orientation of molecule $k$ are denoted by $r_{k}$ and $\omega_{k}$ respectively; they are collectively represented by the shorthand notation $(k)$. Orientations are usually specified by the Euler angles ${ }^{7}$; then $\omega_{k}=\left(\theta_{k}\right.$, $\left.\phi_{k}, \psi_{k}\right)$ and $\Omega \equiv \int d \omega_{k}=8 \pi^{2}$. Let $\mathrm{e}_{\alpha k}(\alpha=1,2,3)$ be a set of orthogonal unit vectors rigidly affixed to molecule $k$. The $e_{\alpha k}$ are defined similarly for all the molecules, so that if molecules $j$ and $k$ can be made coincident by a translation alone then $\mathbf{e}_{\alpha j}=\mathbf{e}_{\alpha k}$. The dipole moment of molecule $k$ can then be expressed as

$$
\mu_{k}=\sum_{\alpha} \mu_{\alpha} e_{\alpha k}
$$

where $\mu_{\mathrm{a}}$ is simply the component of $\mu$ along the $\alpha$ axis in the molecular frame. The magnitude of the molecular dipole moment is

$$
\mu_{0}=\left(\sum_{\alpha} \mu_{\alpha}^{2}\right)^{1 / 2} \text {. }
$$

Imagine that the sample is subjected to an externally applied field that adds the term

$$
\sum_{k} \phi(k)
$$

to the total potential energy of the system. The linear (first-order) deviation $\delta n(1)$ of the single-molecule generic distribution function $n(1)$ from its zero-field value of $\rho / \Omega$ then satisfies ${ }^{3}$

$$
-\beta \phi(1)=(\Omega / \rho) \delta n(1)-\int d(2) \delta n(2) c(12),
$$

where $c(12)$ is the direct correlation function ${ }^{4}$ of the system in zero applied field, and $\beta=(k T)^{-1}$. The spatial part of the integral $\int d(2)$ of course extends only over the sample volume $V$. Equation (3) is our basic starting equation, just as it was for linear molecules. ${ }^{3}$

The external field is taken to be a position-dependent electric field $\mathbf{E}_{0}(\mathbf{r})$, so that 


$$
\phi(1)=-\mu_{1} \cdot E_{0}\left(r_{1}\right)=-\sum_{\alpha} \mu_{\alpha} \mathbf{e}_{\alpha 1} \cdot E_{0}\left(r_{1}\right) .
$$

The macroscopic response of the system to the field is measured by the polarization (dipole moment per unit volume), which is given by

$$
\mathbf{P}\left(\mathbf{r}_{1}\right)=\int d \omega_{1} \mu_{1} \delta n(1)=\sum_{\alpha} \mu_{\alpha} \mathbf{Q}_{\alpha}\left(\mathbf{r}_{1}\right)
$$

where

$$
\mathbf{Q}_{\alpha}\left(\mathbf{r}_{1}\right)=\int d \omega_{1} \mathbf{e}_{\alpha 1} \delta n(1)
$$

We now substitute Eq. (4) into Eq. (3), multiply the resulting equation by $e_{\beta 1}$, and integrate over $\omega_{1}$. The result is

$$
\frac{1}{3} \beta \Omega \mu_{\beta} \mathbf{E}_{0}\left(\mathbf{r}_{1}\right)=(\Omega / \rho) \mathbf{Q}_{\beta}\left(\boldsymbol{r}_{1}\right)-\int d(2) d \omega_{1} \delta n(2) c(12) \mathbf{e}_{\beta 1},
$$

where use has been made of the easily-verified relation

$$
\int d \omega_{k} \mathbf{e}_{\alpha k} \mathbf{e}_{\beta k}=\frac{1}{3} \Omega \delta_{\alpha \beta} \mathbf{U}
$$

in which $\delta_{\alpha \beta}$ is the Kronecker delta and $\mathbf{U}$ is the unit dyadic. Although it is clear from the context, we emphasize that when $\beta$ appears as a subscript it simply represents 1,2 , or 3 and is unrelated to the temperature.

To proceed further something must be known about the direct correlation function $c(12)$. Fortunately something is; a number of independent treatments (most of which were cited in Ref. 3) imply that $c$ (12) becomes asymptotic to $-\beta \phi_{d}(12)$ at long range, where $\phi_{d}(12)$ is the dipole-dipole potential. We can therefore write

$$
c(12)=c_{s}(12)+\beta \mu_{1} \cdot T_{8}\left(\mathbf{r}_{12}\right) \cdot \mu_{2},
$$

where $c_{s}(12)$ is a short-ranged function of (12), $\mathbf{r}_{12}$ $=\mathbf{r}_{1}-\mathbf{r}_{2}, \mathbf{T}_{0}(\mathbf{r})=H(|\mathbf{r}|-\delta) \nabla \nabla|\mathbf{r}|^{-1}$, and $H(x)$ is unity if $x \geqslant 0$ and zero otherwise. It is understood that the limit $\delta-0$ is to be taken after the performance of any spatial integral in which $T_{8}(\mathbf{r})$ appears. Combining Eqs. (7) and (9) and making use of Eqs. (1), (8), and (5), we obtain

$$
\frac{1}{3} \beta \Omega \mu_{\beta} \mathbf{E}_{L}\left(\mathbf{r}_{1}\right)=(\Omega / \rho) \mathbf{Q}_{\beta}\left(\mathbf{r}_{1}\right)-\int d(2) d \omega_{1} \delta n(2) c_{s}(12) \mathbf{e}_{\beta 1},
$$

where the Lorentz electric field $\mathbf{E}_{L}(\mathbf{r})$ is defined by

$$
\mathbf{E}_{L}\left(\mathbf{r}_{1}\right)=\mathbf{E}_{0}\left(\mathbf{r}_{1}\right)+\int d \mathbf{r}_{2} \mathbf{T}_{0}\left(\mathbf{r}_{12}\right) \cdot \mathbf{P}\left(\mathbf{r}_{2}\right)
$$

Since $c_{s}(12)$ is short-ranged, the integral over $r_{2}$ in Eq. (10) can be extended over all space instead of just over the volume $V$.

We restrict attention to external fields $\mathbf{E}_{0}(\mathbf{r})$ that vary slowly with $\mathbf{r}$ in a molecular sense, i.e., compared to the range of $c_{s}(12)$. Then $\delta n(2)$ will also vary slowly with $r_{2}$, so that Eq. (10) may be rewritten as

$$
\frac{1}{3} \beta \Omega \mu_{\beta} \mathbf{E}_{L}\left(\mathbf{r}_{1}\right)=(\Omega / \rho) Q_{\beta}\left(\mathbf{r}_{1}\right)
$$

$$
-\int d \omega_{2} \delta n\left(\mathbf{r}_{1}, \omega_{2}\right) \int d \mathbf{r}_{2} d \omega_{1} c_{s}(12) \mathbf{e}_{\beta 1}
$$

Now assume that $c_{s}(12)$ depends only on relative positions and orientations of molecules 1 and 2 . [Since $c_{s}(12)$ is a short-ranged function, this assumption is very reasonable even though the system is finite.] Then it is clear that the inner integral in $\mathrm{Eq}_{\text {. }}$ (12) can be expressed as a linear combination of the vectors $\mathrm{e}_{\alpha 2}$,

$$
\int d \mathrm{r}_{2} d \omega_{1} c_{s}(12) \mathbf{e}_{\beta 1}=\Omega \sum_{\alpha} c_{\beta \alpha} \mathbf{e}_{\alpha 2},
$$

where the coefficients $c_{\beta_{\alpha}}$ are independent of $r_{1}$ and $\omega_{2}$. Since the $\theta_{\alpha 2}$ are orthonormal, these coefficients are given by

$$
c_{\alpha \beta}=\Omega^{-2} \int d \mathbf{r}_{2} d \omega_{1} d \omega_{2} c_{s}(12) \mathbf{e}_{\alpha 1} \cdot \mathbf{e}_{\beta 2},
$$

where we have performed a further average over $\omega_{2}$ to make the result look more symmetrical. Since all the molecules are identical, $c_{s}(12)$ is symmetric and hence $c_{\alpha \beta}=c_{\beta \alpha}$.

Combining Eqs. (12) and (13), we obtain

$$
\frac{1}{3} \beta \rho \mu_{\beta} \mathbf{E}_{L}\left(\mathbf{r}_{1}\right)=\mathbf{Q}_{B}\left(\mathbf{r}_{1}\right)-\rho \sum_{\alpha} c_{\beta \alpha} \mathbf{Q}_{\alpha}\left(\mathbf{r}_{1}\right) .
$$

Let $\hat{\mu}$ be the column vector with elements $\mu_{\alpha}, \hat{Q}(\mathbf{r})$ be the column vector with elements $Q_{\alpha}(r)$, and $\hat{C}$ be the $3 \times 3$ symmetric matrix with elements $c_{\alpha \beta}$. Then Eq. (15) can be written in matrix form,

$$
\hat{Q}\left(r_{1}\right)-\rho \hat{C} \hat{Q}\left(r_{1}\right)=\frac{1}{3} \beta \rho E_{L}\left(r_{1}\right) \hat{\mu},
$$

which has the formal solution

$$
\hat{Q}\left(\mathbf{r}_{1}\right)=\frac{1}{3} \beta \rho \mathrm{E}_{L}\left(\mathbf{r}_{1}\right)(\hat{I}-\rho \hat{C})^{-1} \hat{\mu},
$$

in which $\hat{I}$ denotes the $3 \times 3$ unit matrix with elements $\delta_{\alpha \beta}$. Now the polarization, given by Eq. (5), can also be written in matrix form,

$$
\mathbf{P}\left(\mathbf{r}_{1}\right)=\hat{\mu}^{\mathrm{T}} \hat{Q}\left(\mathbf{r}_{1}\right) \text {, }
$$

where $\hat{\mu}^{T}$ is the row vector corresponding to the column vector $\hat{\mu}$. Combining Eqs. (17) and (18), we obtain

$$
\mathbf{P}\left(\mathbf{r}_{1}\right)=\frac{1}{3} \Leftrightarrow \rho\left[\hat{\mu}^{T}(\hat{I}-\rho \hat{C})^{-1} \hat{\mu}\right] \mathbf{E}_{L}\left(r_{1}\right) \text {. }
$$

Therefore, ${ }^{2}$ the dielectric constant exists and is given by

$$
\begin{aligned}
(\epsilon-1) /(\epsilon+2) & =(4 \pi / 9) \beta \rho \hat{\mu}^{T}(\hat{I}-\rho \hat{C})^{-1} \hat{\mu} \\
& =\frac{4 \pi}{9} \beta \rho \sum_{\alpha \beta} \mu_{\alpha} \mu_{\beta}\left[(\hat{I}-\rho \hat{C})^{-1}\right]_{\alpha \beta} .
\end{aligned}
$$

Since the matrix $(\hat{I}-\rho \hat{C})$ is only $3 \times 3$, it can be inverted fairly easily. This will not be done, however, as it has no advantage for our purposes.

To complete the development we show that $c_{s}(12)$ may be replaced by $c(12)$ in Eq. (14) without changing $c_{\alpha \beta}$. To do so, it suffices to show that the quantity

$$
d_{\alpha \beta}(\mathbf{r})=\int d \omega_{1} d \omega_{2}\left[\mu_{1} \cdot \mathbf{T}_{0}(\mathbf{r}) \cdot \mu_{2}\right]\left(\mathbf{e}_{\alpha 1} \cdot \mathbf{e}_{\beta 2}\right)
$$

vanishes. Using Eqs. (1) and (8), it is easy to show that $d_{\alpha \beta}$ reduces to 


$$
d_{\alpha \beta}(r)=(\Omega / 3)^{2} \mu_{\alpha} \mu_{\beta} \mathbf{T}_{\delta}(\mathbf{r}): \mathbf{U}
$$

But

$\mathbf{T}_{\delta}(\mathbf{r}): \mathbf{U}=H(|\mathbf{r}|-\delta) \nabla^{2}|\mathbf{r}|^{-1}=-4 \pi H(|\mathbf{r}|-\delta) \delta_{D}(\mathbf{r})$,

where $\delta_{D}(\mathbf{r})$ is the Dirac delta function. This clearly vanishes for all $\mathbf{r}$; thus $d_{\alpha \beta}(\mathbf{r})=0$ and $c_{\alpha \beta}$ can be computed from the alternative expression

$$
c_{\alpha \beta}=\Omega^{-2} \int d \mathrm{r}_{2} d \omega_{1} d \omega_{2} c(12) \mathbf{e}_{\alpha 1} \cdot \mathbf{e}_{\beta 2},
$$

where the integral over $r_{2}$ can be extended over all space, just as in Eqs. (12) -(14).

\section{THE FORM OF $\epsilon$}

We now wish to consider Eq. (20) for $\epsilon$ in greater detail. First we verify that Eq. (20) reduces properly to the form appropriate to axially symmetric molecules. ${ }^{3}$ Let the molecular axis $\alpha=3$ be the symmetry axis, so that $\mu_{1}=\mu_{2}=0$ and $\mu_{3}=\mu_{0}$. The orientation of the symmetry axis of molecule $k$ is specified by $\omega_{k}^{\prime}=\left(\theta_{k}, \phi_{k}\right)$ and the angle of rotation about this axis is denoted by $\psi_{k}$. Thus $\omega_{k}=\left(\omega_{k}^{\prime}, \psi_{k}\right)$ znd $d \omega_{k}=d \omega_{k}^{\prime} d \psi_{k}$. Clearly $c(12)$ is independent of $\psi_{1}$ and $\psi_{2}$, so that Eq. (24) becomes

$c_{\alpha \beta}=\left(8 \pi^{2}\right)^{-2} \int d \mathrm{r}_{2} d \omega_{1}^{\prime} d \omega_{2}^{\prime} c(12)\left(\int d \psi_{1} \mathbf{e}_{\alpha 1}\right) \cdot\left(\int d \psi_{2} \mathbf{e}_{B 2}\right)$.

But

$$
\int d \psi_{k} \mathbf{e}_{\alpha k}=2 \pi \delta_{\alpha 3} \mathbf{e}_{3 k}
$$

therefore,

$$
c_{\alpha \beta}=\delta_{\alpha 3} \delta_{\beta B} B=\delta_{\alpha \beta} \delta_{\alpha 3} B,
$$

where

$$
B=(4 \pi)^{-2} \int d \mathbf{r}_{2} d \omega_{1}^{\prime} d \omega_{2}^{\prime} c(12) \mathbf{e}_{31} \cdot \mathbf{e}_{32} .
$$

Since the only nonzero element of $\hat{C}$ is $c_{3 s},(\hat{I}-\rho \hat{C})$ is a diagonal matrix and its inverse can be computed trivially. The result is

$$
\left[(\hat{I}-\rho \hat{C})^{-1}\right]_{\alpha \beta}=\delta_{\alpha \beta}\left(1-\rho \delta_{\alpha 3} B\right)^{-1} .
$$

Equation (20) then becomes

$$
\begin{aligned}
(\epsilon-1) /(\epsilon+2) & =(4 \pi / 9) \beta \rho \mu_{0}^{2}\left[(\hat{I}-\rho \hat{C})^{-1}\right]_{33} \\
& =(4 \pi / 9) \beta \rho \mu_{0}^{2}(1-\rho B)^{-1},
\end{aligned}
$$

which is just the result obtained earlier under the restriction of axial symmetry. ${ }^{2,3}$

We next compare Eq. (20) with the result derived by $\mathrm{H} \phi y e$ and Stell (HS) ${ }^{5}$ in their Appendix A. To perform this comparison it is necessary to express the $c_{\alpha \beta}$ in terms of the Euler angles. Let $a_{\alpha}$ be a set of orthogonal unit vectors fixed in the laboratory, and let the Euler angles $\left(\theta_{k}, \phi_{k}, \psi_{k}\right)$ correspond to the rotation that transforms the $a_{\alpha}$ into the $e_{\alpha k}$. This transformation is performed using the rotation matrix $\hat{A}$, whose elements $A_{\alpha \beta}$ are functions of the Euler angles of the molecule in question:

$$
\mathbf{e}_{\alpha k}=\sum_{\beta} A_{\alpha \beta}(k) \mathrm{a}_{\beta} .
$$

The matrix $\hat{A}$ is given by Eq. (4-46) of Goldstein?; it is just the transpose of the matrix $M_{1}$ defined by Eq. (A5) of HS, whose elements they denote by $f_{\alpha \beta}^{1}$. Thus $A_{\alpha \beta}(k)=f_{\beta \alpha}^{1}(k)$, which together with Eq. (31) implies that

$$
\mathbf{e}_{\alpha 1} \cdot \mathbf{e}_{\beta 2}=\sum_{\gamma} f_{\gamma \alpha}^{1}(1) f_{\gamma \beta}^{1}(2)
$$

Equation (14) can therefore be rewritten as

$$
c_{\alpha \beta}=\Omega^{-2} \int d \mathrm{r}_{2} d \omega_{1} d \omega_{2} c_{s}(12) \sum_{\gamma} f_{\gamma \alpha}^{1}(1) f_{\gamma \beta}^{1}(2) \text {. }
$$

Now it follows from Eqs. (A7)-(A9) of HS that, in their notation,

$$
c_{i j}^{n}=\left(\rho / \Omega^{2}\right) \int d r_{2} d \Omega_{1} d \Omega_{2} c_{0}(12) \sum_{k} f_{k i}^{n}(1) f_{k j}^{n}(2) .
$$

Since our $c_{s}(12)$ is the same as HS's $c_{0}(12)$, we see that our $\rho c_{\alpha \beta}$ is the same as their $c_{\alpha \beta}^{1}$. This equivalence enables us to write their expression for $\epsilon$ [obtained by combining their Eqs. (2.12a), (A13), (A15), and (A16)] in our notation as

$$
(\epsilon-1) /(\epsilon+2)=(4 \pi / 9) \beta \rho \mu_{0}^{2}\left[(\hat{I}-\rho \hat{C})^{-1}\right]_{33} \text {. }
$$

But HS have assumed, in effect, that the molecular coordinate system has been chosen so that the dipole moment lies along the molecular axis $\alpha=3$. When Eq. (20) is specialized to this choice, we obtain precisely Eq. (35). We thereby confirm the result of HS by an entirely different method. However, Eq. (20) is more general in mathematical form then the HS expression, since we allow an arbitrary choice of the molecular coordinate system. Moreover, since our expression for $c_{\alpha \beta}$ involves the $e_{\alpha k}$ directly it is independent of the choice of orientational coordinates, thereby allowing the use of descriptions other then the Euler angles if desired.

Finally, we show that the inverse of the matrix $(\hat{I}-\rho \hat{C})$ can be directly expressed in terms of the shortrange total correlation function $h_{0}(12)$ defined by

$$
h_{0}(12)=c_{s}(12)+(\rho / \Omega) \int d(3) c_{s}(13) h_{0}(32) \text {. }
$$

Multiply Eq. (36) by $e_{\alpha 1} \cdot e_{\beta 2}$ and integrate over $r_{2}, \omega_{1}$, and $\omega_{2}$. After manipulations similar to those performed earlier, one obtains

$$
h_{\alpha \beta}=c_{\alpha \beta}+\rho \sum_{\gamma} c_{\alpha \gamma} h_{\gamma \beta},
$$

where

$$
h_{\alpha \beta}=\Omega^{-2} \int d \mathbf{r}_{2} d \omega_{1} d \omega_{2} h_{0}(12) \mathbf{e}_{\alpha 1} \cdot \mathbf{e}_{\beta 2} .
$$

Let $\hat{H}$ denote the matrix with elements $h_{\alpha \beta}$; then Eq.

(37) can be written in matrix form as

$$
\hat{H}=\hat{C}+\rho \hat{C} \hat{H} \text {. }
$$

Multiply this equation by $\rho$ and add $\hat{I}$ to both sides. Then after rearrangement one finds

$$
(\hat{I}-\rho \hat{C})(\hat{I}+\rho \hat{H})=\hat{I},
$$


so that

$$
(\hat{I}-\rho \hat{C})^{-1}=(\hat{I}+\rho \hat{H}) .
$$

Substitution of Eq. (41) into Eq. (20) then yields

$$
\begin{aligned}
\frac{\epsilon-1}{\epsilon+2} & =\frac{4 \pi}{9} \beta \rho \sum_{\alpha \beta} \mu_{\alpha} \mu_{\beta}\left(\delta_{\alpha \beta}+\rho h_{\alpha \beta}\right) \\
& =\frac{4 \pi}{9} \beta \rho\left[\mu_{0}^{2}+\rho \Omega^{-2} \int d \mathbf{r}_{2} d \omega_{1} d \omega_{2} h_{0}(12) \mu_{1} \circ \mu_{2}\right]
\end{aligned}
$$

where Eqs. (1) and (2) have been used. This expression is identical to the result of combining Eqs. (2.12a), $(2.12 b)$, and $(2.12 \mathrm{e})$ of $\mathrm{H} \phi$ ye and Stell, ${ }^{5}$ since our function $h_{0}(12)$ is the same as their function $(\Omega / \rho)^{2} W(12)$. Conversely, if one begins with $\mathrm{Eq}$. (42) the relation (41) provides the simplest route to $\mathrm{Eq}$. (20).

\section{SUMMARY}

We have shown that if the direct correlation function is of the form given in Eq. (9), where $c_{s}(12)$ is a short- ranged function that depends only on relative positions and orientations of molecules 1 and 2 , then $\epsilon$ exists and is given by $\mathrm{Eq}$. (20). Equation (20) is identical in physical content to the expression for $\epsilon$ in terms of $c(12)$ derived earlier by Høye and Stell, but is more general in mathematical form. The matrix inverse appearing in Eq. (20) was found to be simply related, via Eq. (41), to the short-range total correlation function conjugate to $c_{s}(12)$.

${ }^{1}$ J. D. Ramshaw, J. Chem. Phys. 55, 1763 (1971).

2J. D. Ramshaw, J. Chem. Phys. 57, 2684 (1972).

${ }^{3}$ J. D. Ramshaw, J. Chem. Phys. 66, 3134 (1977).

${ }^{4} \mathrm{G}$. S. Rushbrooke, in Physics of Simple Liquids, edited by H. N. V. Temperley et al. (Wiley-Interscience, New York, 1968), p. 25.

${ }^{5} J$. S. Hфye and G. Stell, J. Chem. Phys. 64, 1952 (1976).

${ }^{6}$ J. S. Hфye and G. Stell, J. Chem. Phys. 61, 562 (1974).

${ }^{7} \mathrm{H}$. Goldstein, Classical Mechanics (Addison-Wesley, Reading, 1950). 